\title{
Multivalently meromorphic functions with two fixed points defined by Srivastava-Attiya operator
}

\author{
K. Vijaya* and M. Kasthuri \\ School of Advanced Sciences, VIT University, Vellore 632014,India \\ *Corresponding author Email: kvijaya@vit.ac.in
}

Copyright (C)2014 K. Vijaya and M. Kasthuri. This is an open access article distributed under the Creative Commons Attribution License, which permits unrestricted use, distribution, and reproduction in any medium, provided the original work is properly cited.

\begin{abstract}
In the present investigation we define a new class of meromorphic functions on the punctured unit disk $\mathcal{U}^{*}:=\{z \in$ $\mathbb{C}: 0<|z|<1\}$ by making use of the Srivastava-Attiya operator. Coefficient inequalities, growth and distortion inequalities, as well as radii of meromorphically starlikeness are obtained. We also establish some results concerning the convolution products and inclusion results.
\end{abstract}

Keywords: Convolution(Hadamard product),Hurwitz-Learch zeta function, Integral operator, Meromorphic functions,Starlike function, Convex function,

2010 AMS Subject Classification:Primary 30C45; Secondary 30C50

\section{Introduction}

Let $\mathcal{M}_{p}$ denote the class of functions of the form

$$
f(z)=\frac{a_{p}}{z^{p}}+\sum_{n=1}^{\infty} a_{n} z^{n-p},\left(a_{n} \geq 0 ; p \in \mathbb{N}=\{1,2,3 \ldots\}\right),
$$

which are meromorphic and p-valent in the punctured unit disc

$$
\mathcal{U}^{*}=\{z \in \mathbb{C}: 0<|z|<1\}
$$

Let $g(z)=\frac{b_{p}}{z^{p}}+\sum_{n=1}^{\infty} b_{n} z^{n-p},\left(b_{n} \geq 0 ; p \in \mathbb{N}=\{1,2,3 \ldots\}\right)$, then the Convolution (or Hadamard) product of $f(z)$ and $g(z)$ is defined as

$$
f(z) * g(z)=(f * g)(z)=\frac{a_{p} b_{p}}{z^{p}}+\sum_{n=1}^{\infty} a_{n} b_{n} z^{n-p}, \quad\left(a_{n}, b_{n} \geq 0 ; p \in \mathbb{N}=\{1,2,3 \ldots\}\right) .
$$

Now, we recall a general Hurwitz-Lerch Zeta function $\Phi(z, s, a)$ defined by

$$
\Phi(z, s, a):=\sum_{k=0}^{\infty} \frac{z^{k}}{(k+a)^{s}}
$$


where $z \in \mathcal{U}=z:|z|<1$ and $a \in \mathbb{C} \backslash\left\{\mathbb{Z}_{0}^{-}\right\} ; s \in \mathbb{C}, \mathfrak{R}(s)>1$ and $|z|=1$ as usual $\mathbb{Z}_{0}^{-}:=\mathbb{Z} \backslash\{\mathbb{N}\} ;(\mathbb{Z}:=$ $\{0, \pm 1, \pm 2, \pm 3, \ldots\} ; \mathbb{N}:=\{1,2,3, \ldots\})$. Several interesting properties and characteristics of the Hurwitz-Lerch Zeta function $\Phi(z, s, a)$ can be found in the recent investigations by Choi and Srivastava [8], Ferreira and Lopez [10], Garg et al. [12], Lin and Srivastava [13], Lin et al. [14], and others.

For the class of analytic functions denote by $\mathcal{A}$ consisting of functions of the form

$$
f(z)=z+\sum_{n=2}^{\infty} a_{n} z^{n}, \quad z \in \mathcal{U}
$$

Srivastava and Attiya [34] introduced and investigated the linear operator

$$
\mathcal{J}_{b}^{s}: \mathcal{A} \rightarrow \mathcal{A}
$$

defined in terms of the Hadamard product (or convolution) by

$$
\mathcal{J}_{b}^{s} f(z)=G_{b, s} * f(z)
$$

where $z \in \mathcal{U} ; b \in \mathbb{C} \backslash\left\{\mathbb{Z}_{0}^{-}\right\} ; s \in \mathbb{C}$, for convenience,we write

$$
G_{s, b}(z):=(1+b)^{s}\left[\Phi(z, s, b)-b^{-s}\right] \quad(z \in \mathcal{U}) .
$$

It is easy to observe from (1), (3) and (4)that

$$
\mathcal{J}_{b}^{s} f(z)=z+\sum_{n=2}^{\infty}\left(\frac{1+b}{n+b}\right)^{s} a_{n} z^{n} .
$$

It is well known that the Srivastava-Attiya operator $\mathcal{J}_{b}^{s}$ contains, among its special cases, the integral operators introduced and investigated earlier by (for example) Alexander [1], Libera [19], Bernardi [6], and Jung et al. [17].

Motivated essentially by the above-mentioned Srivastava-Attiya operator, in this paper for functions $f \in \mathcal{M}_{p}$ we define the operator

$$
\mathcal{J}_{b}^{s}: \mathcal{M}_{p} \rightarrow \mathcal{M}_{p}
$$

by the convolution

$$
\mathcal{J}_{b}^{s} f(z)=G_{b, p}^{s} * f(z) \quad\left(z \in \mathcal{U}^{*} ; b \in \mathbb{C} \backslash\left\{\mathbb{Z}_{0}^{-}\right\} ; s \in \mathbb{C}\right)
$$

for convenience,

$$
G_{b, p}^{s}(z):=(1+b)^{s}\left[\Phi_{p}(z, s, b)-b^{-s}\right] \quad\left(z \in \mathcal{U}^{*}\right)
$$

where

$$
\Phi_{p}(z, s, b)=\frac{1}{b^{s}}+\frac{z^{-p}}{(1+b)^{s}}+\frac{z^{-p+1}}{(2+b)^{s}}+\ldots
$$

For $f(z) \in \mathcal{M}_{p}$ it is easy to observe from the above equations that

$$
\mathcal{J}_{b}^{s} f(z)=z^{-p}+\sum_{n=1}^{\infty} C_{b}^{s}(n) a_{n} z^{n-p}
$$

where

$$
C_{b}^{s}(n)=\left|\left(\frac{1+b}{n+1+b}\right)^{s}\right|
$$

and (throughout this paper unless otherwise mentioned) the parameters $s, b$ are constrained as

$$
b \in \mathbb{C} \backslash\left\{\mathbb{Z}_{0}^{-}\right\} ; s \in \mathbb{C} \text { and } p \in \mathbb{N} .
$$

For multivalently meromorphic functions $f \in \mathcal{M}_{p}$, the normalization

$$
\left.z^{1+p} f(z)\right|_{z=0}=0 \quad \text { and }\left.\quad z^{p} f(z)\right|_{z=0}=1
$$


is classical. One can obtain interesting results by applying Montel's normalization [25] of the form

$$
\left.z^{1+p} f(z)\right|_{z=0}=0 \quad \text { and }\left.\quad z^{p} f(z)\right|_{z=\rho}=1
$$

where $\rho$ is a fixed point from the unit disk $\mathcal{U}^{*}$. Note that if $\rho=0$ the normalization 11 is the classical normalization 10 .

Meromorphic multivalent functions have been extensively studied by (for example) Mogra [23], Uralegaddi and Ganigi[36], Uralegaddi and Somanatha [37], Aouf [2], Aouf and Hossen[3],

Srivastava et al.[35], Owa et al. [27], Joshi and Aouf [15], Joshi and Srivastava [16],Aouf et al.[4],Raina and Srivastava [30], Yang [39], Kulkarni et al.[18],Liu [22] and Liu and Srivastava [20]and [21]). Motivated by the works of Vijaya et. al. [38],we define the following new subclass $\mathcal{M}_{b}^{s}(\alpha, \beta)$ of meromorphic starlike functions in the parabolic region of functions in $\mathcal{M}_{p}$ by making use of the generalized operator $\mathcal{J}_{b}^{s}$ with Montel's normalization, to study its characteristic properties (for example coefficient inequalities, growth and distortion inequalities, radii of starlikeness. We also establish some results concerning the convolution products).

For fixed parameters $\alpha \geq \frac{1}{2+\beta} ; 0 \leq \beta<1$, we let $\mathcal{M}_{b}^{s}(\alpha, \beta)$ the meromorphically $p$-valent function $f \in \mathcal{M}_{p}$ with two fixed points (or classical normalization) if it satisfies the following

$$
\left|\frac{z\left(\mathcal{J}_{b}^{s} f(z)\right)^{\prime}}{p\left(\mathcal{J}_{b}^{s} f(z)\right)}+\alpha+\alpha \beta\right| \leq \Re\left\{\frac{-z\left(\mathcal{J}_{b}^{s} f(z)\right)^{\prime}}{p\left(\mathcal{J}_{b}^{s} f(z)\right)}+\alpha-\alpha \beta\right\},\left(n \in \mathbb{N}_{0}\right)
$$

where $\mathcal{J}_{b}^{s} f(z)$ given by $(8)$.

Further ,we let the subclass $\mathcal{M}_{b}^{s}(\alpha, \beta, \rho)$ satisfying the condition (12) with Montel's normalization (11).

In the following section we discuss certain characterization properties for $f \in \mathcal{M}_{b}^{s}(\alpha, \beta)$.

\section{Properties of the class $\mathcal{M}_{b}^{s}(\alpha, \beta)$}

Theorem 2.1. Let $f \in \mathcal{M}_{p}$, then $f$ is in the class $\mathcal{M}_{b}^{s}(\alpha, \beta)$ if and only if

$$
\sum_{n=1}^{\infty} d_{n}\left|a_{n}\right| \leq p(1-\alpha \beta) a_{p}
$$

where

$$
d_{n}=[n-p(1-\alpha \beta)] C_{b}^{s}(n)
$$

and

$$
\alpha>\frac{1}{2+\beta} ; \quad 0 \leq \beta<1 ; p \in \mathbb{N}, n \in \mathbb{N}_{0}
$$

Proof. Suppose that $f \in \mathcal{M}_{b}^{s}(\alpha, \beta)$, then by the inequality(12), we have

$$
\left|\frac{z\left(\mathcal{J}_{b}^{s} f(z)\right)^{\prime}}{p\left(\mathcal{J}_{b}^{s} f(z)\right)}+\alpha+\alpha \beta\right| \leq \Re\left\{\frac{-z\left(\mathcal{J}_{b}^{s} f(z)\right)^{\prime}}{p\left(\mathcal{J}_{b}^{s} f(z)\right)}+\alpha-\alpha \beta\right\},\left(n \in \mathbb{N}_{0}\right)
$$

that is,

$$
\begin{aligned}
\Re\left\{\frac{z\left(\mathcal{J}_{b}^{s} f(z)\right)^{\prime}}{p\left(\mathcal{J}_{b}^{s} f(z)\right)}+\alpha+\alpha \beta\right\} & \leq\left|\frac{z\left(\mathcal{J}_{b}^{s} f(z)\right)^{\prime}}{p\left(\mathcal{J}_{b}^{s} f(z)\right)}+\alpha+\alpha \beta\right| \\
& \leq \Re\left\{\frac{-z\left(\mathcal{J}_{b}^{s} f(z)\right)^{\prime}}{p\left(\mathcal{J}_{b}^{s} f(z)\right)}+\alpha-\alpha \beta\right\} .
\end{aligned}
$$

Hence,

$$
\Re\left\{\frac{z\left(\mathcal{J}_{b}^{s} f(z)\right)^{\prime}}{p\left(\mathcal{J}_{b}^{s} f(z)\right)}+\alpha \beta\right\} \leq 0 .
$$


Substituting for $\mathcal{J}_{b}^{s} f(z)$ and $\left(\mathcal{J}_{b}^{s} f(z)\right)^{\prime}$, we get

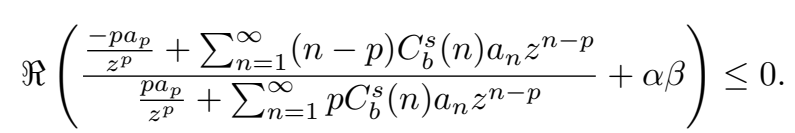

Since $\Re(z) \leq|z|$, we have

$$
\left|-p a_{p}+\sum_{n=1}^{\infty}(n-p) C_{b}^{s}(n) a_{n} z^{n}+p a_{p} \alpha \beta+p \alpha \beta \sum_{n=1}^{\infty} C_{b}^{s}(n) a_{n} z^{n}\right| \leq 0
$$

and by letting $|z| \rightarrow 1^{-}$, we get

$$
\sum_{n=1}^{\infty}[n-p(1-\alpha \beta)] C_{b}^{s}(n)\left|a_{n}\right| \leq p(1-\alpha \beta) a_{p} .
$$

In order to prove the converse, we assume that the inequality holds true.Then, if we let $z \in \partial \mathcal{U}$, we find from 1 and 13 , that

$$
\begin{aligned}
\Re\left\{\frac{z\left(\mathcal{J}_{b}^{s} f(z)\right)^{\prime}}{p\left(\mathcal{J}_{b}^{s} f(z)\right)}+\alpha \beta\right\} \leq & 0 . \\
& \Re\left(\frac{\frac{-p a_{p}}{z^{p}}+\sum_{n=1}^{\infty}(n-p) C_{b}^{s}(n) a_{n} z^{n-p}}{\frac{p a_{p}}{z^{p}}+\sum_{n=1}^{\infty} p C_{b}^{s}(n) a_{n} z^{n-p}}+\alpha \beta\right) \leq 0 .
\end{aligned}
$$

Since $\Re(z) \leq|z|$, we have

$$
\sum_{n=1}^{\infty} \frac{[n-p(1-\alpha \beta)] C_{b}^{s}(n)\left|a_{n}\right|}{p(1-\alpha \beta) a_{p}} \leq 1 .
$$

which completes the proof.

For the sake of brevity throughout this paper, we let

$$
d_{n}=[n-p(1-\alpha \beta)] C_{b}^{s}(n)
$$

and

$$
\alpha>\frac{1}{2+\beta} ; \quad 0 \leq \beta<1 ; p \in \mathbb{N}, n \in \mathbb{N}_{0}
$$

unless otherwise state.

Theorem 2.2. (Coefficient Estimate) Let $f \in \mathcal{M}_{b}^{s}(\alpha, \beta)$, then

$$
\sum_{n=1}^{\infty}\left|a_{n}\right| \leq \frac{p(1-\alpha \beta) a_{p}}{d_{n}} .
$$

Theorem 2.3. Let $f \in \mathcal{M}_{b}^{s}(\alpha, \beta, \rho)$, then

$$
\sum_{n=1}^{\infty}\left|a_{n}\right| \leq \frac{p(1-\alpha \beta)}{d_{n}+p(1-\alpha \beta) \rho^{n}}
$$

Proof. Let $f \in \mathcal{M}_{b}^{s}(\alpha, \beta, \rho)$. Since $f \in \mathcal{M}_{b}^{s}(\alpha, \beta)$ by Theorem 2.1, we have

$$
\sum_{n=1}^{\infty} d_{n}\left|a_{n}\right| \leq p(1-\alpha \beta) a_{p}
$$

For $f \in \mathcal{M}_{p}$, by Montel's normalization (11),we have

$$
\left.z^{p}\left(a_{p} z^{-p}+\sum_{n=1}^{\infty} a_{n}^{n-p}\right)\right|_{z=\rho}=1
$$




$$
a_{p}=1-\sum_{n=1}^{\infty} a_{n} \rho^{n}
$$

Therefore from (13), we have

$$
\begin{aligned}
& \sum_{n=1}^{\infty} d_{n}\left|a_{n}\right| \leq p(1-\alpha \beta)\left(1-\sum_{n=1}^{\infty} a_{n} \rho^{n}\right) . \\
& \sum_{n=1}^{\infty}\left[d_{n}+p(1-\alpha \beta) \rho^{n}\right]\left|a_{n}\right| \leq p(1-\alpha \beta) .
\end{aligned}
$$

Hence

$$
\sum_{n=1}^{\infty}\left|a_{n}\right| \leq \frac{p(1-\alpha \beta)}{d_{n}+p(1-\alpha \beta) \rho^{n}}
$$

Theorem 2.4. (Distortion Bounds) If $f \in \mathcal{M}_{b}^{s}(\alpha, \beta, \rho)$, then

$$
\left(\frac{d_{1}-p(1-\alpha \beta) r}{d_{1}+p(1-\alpha \beta) \rho}\right) r^{-p} \leq|f(z)| \leq\left(\frac{d_{1}+p(1+\alpha \beta) r}{d_{1}+p(1-\alpha \beta) \rho}\right) r^{-p},(0<|z|=r<1) .
$$

Proof. Let $f \in \mathcal{M}_{b}^{s}(\alpha, \beta)$. Then we find from Theorem 2.3 , that

$$
\sum_{n=1}^{\infty}\left[d_{n}+p(1-\alpha \beta) \rho^{n}\right]\left|a_{n}\right| \leq p(1-\alpha \beta)
$$

which yields,

$$
\sum_{n=1}^{\infty}\left|a_{n}\right| \leq \frac{p(1-\alpha \beta)}{\left[d_{1}+p(1-\alpha \beta) \rho\right]}
$$

We have

$$
\begin{aligned}
& |f(z)|=\left|a_{p} z^{-p}+\sum_{n=1}^{\infty} a_{n} z^{n-p}\right| \\
& \leq\left(1-\sum_{n=1}^{\infty}\left|a_{n}\right| \rho^{n}+\sum_{n=1}^{\infty}\left|a_{n}\right| r^{n}\right) r^{-p} \\
& \leq\left(1-(\rho-r) \sum_{n=1}^{\infty}\left|a_{n}\right|\right) r^{-p} \\
& \leq\left(\frac{d_{1}+p(1-\alpha \beta) r}{d_{1}+p(1-\alpha \beta) \rho}\right) r^{-p} .
\end{aligned}
$$

On the other hand we have,

$$
|f(z)| \geq\left(\frac{d_{1}-p(1-\alpha \beta) r}{d_{1}+p(1-\alpha \beta) \rho}\right) r^{-p}
$$

Hence the proof.

Using classical normalization, ( that is by taking $\rho=0$ ) we state the following distortion result without proof.

Theorem 2.5. If $f \in \mathcal{M}_{b}^{s}(\alpha, \beta)$, then

$$
\left(1-\frac{p(1-\alpha \beta) r}{d_{1}}\right) r^{-p} \leq|f(z)| \leq\left(1+\frac{p(1+\alpha \beta) r}{d_{1}}\right) r^{-p},(0<|z|=r<1) .
$$




\section{The Radii of Meromorphically Starlikeness}

Theorem 3.1. Let the function $f(z)$ defined by (1) be in the class $\mathcal{M}_{b}^{s}(\alpha, \beta)$, then we have $f(z)$ is meromorphically p-valent starlike of order $\mu(0 \leq \mu<p)$ in the disc $|z|<r_{1}$, that is,

$$
\Re\left(-\frac{z f^{\prime}(z)}{f(z)}\right)>\mu, \quad|z|<r_{1} ; 0 \leq \mu<p ; p \in \mathbb{N},
$$

where

$$
|z| \leq\left(\frac{d_{n}(p-\mu)}{p(n-p+\mu)(1-\alpha \beta)}\right)^{\frac{1}{n}}
$$

Proof. Let $f(z)=a_{p} z^{-p}+\sum_{n=1}^{\infty} a_{n} z^{n-p}$. Then we easily get

$$
\left|\frac{\frac{z f^{\prime}(z)}{f(z)}+p}{\frac{z f^{\prime}(z)}{f(z)}-p+2 \mu}\right| \leq \frac{\sum_{n=1}^{\infty} n a_{n}|z|^{n}}{2(p-\mu) a_{p}+\sum_{n=1}^{\infty}(n-2 p+2 \mu) a_{n}|z|^{n}} .
$$

Thus, we have the desired inequality

$$
\left|\frac{\frac{z f^{\prime}(z)}{f(z)}+p}{\frac{z f^{\prime}(z)}{f(z)}-p+2 \mu}\right| \leq 1, \quad \text { if, } \quad \sum_{n=1}^{\infty} \frac{(n-p+\mu)}{|p-\mu| a_{p}}\left|a_{n}\right||z|^{n} \leq 1 .
$$

Since $f \in \mathcal{M}_{b}^{s}(\alpha, \beta)$ from Theorem 2.1 , we have

$$
\sum_{n=1}^{\infty} \frac{d_{n}\left|a_{n}\right|}{p(1-\alpha \beta) a_{p}} \leq 1
$$

From (20) and (21)

$$
\begin{gathered}
\frac{n-p+\mu}{|p-\mu| a_{p}}|z|^{n} \leq\left\{\frac{d_{n}}{p(1-\alpha \beta) a_{p}}\right\} \\
|z| \leq\left\{\frac{d_{n}(p-\mu)}{p(n-p+\mu)(1-\alpha \beta)}\right\}^{\frac{1}{n}} .
\end{gathered}
$$

which completes proof .

\section{Convolution properties}

For the function

$$
f_{j}(z)=a_{p, j} z^{-p}+\sum_{n=1}^{\infty}\left|a_{n, j}\right| z^{n-p}, \quad(j=1,2 ; p \in \mathbb{N})
$$

we denote by $\left(f_{1} * f_{2}\right)(z)$ the Hadamard product( or Convolution) of the functions $f_{1}(z)$ and $f_{2}(z)$, that is,

$$
\left(f_{1} * f_{2}\right)(z)=a_{p, 1} a_{p, 2} z^{-p}+\sum_{n=1}^{\infty}\left|a_{n, 1}\right|\left|a_{n, 2}\right| z^{n-p} .
$$

Theorem 4.1. For the function $f_{j}(z)(j=1,2)$ defined by (22)be in the class $\mathcal{M}_{b}^{s}(\alpha, \beta)$. Then $\left(f_{1} * f_{2}\right)(z) \in$ $\mathcal{M}_{b}^{s}(\alpha, \delta)$ where

$$
\delta \leq \frac{1}{\alpha}\left(1-\frac{p(1-\alpha \beta)^{2} C_{b}^{s}(1)}{p^{2}(1-\alpha \beta)^{2} C_{b}^{s}(1)+d_{1}^{2}}\right)
$$

where $d_{1}=[1-p(1-\alpha \beta)] C_{b}^{s}(1)$ and $C_{b}^{s}(1)=\left|\left(\frac{1+b}{2+b}\right)^{s}\right|$. 
Proof. Let $f_{1}(z)=a_{p, 1} z^{-p}+\sum_{n=1}^{\infty}\left|a_{n, 1}\right| z^{n-p}$ and $f_{2}(z)=a_{p, 2} z^{-p}+\sum_{n=1}^{\infty}\left|a_{n, 2}\right| z^{n-p}$ be in the class $\mathcal{M}_{b}^{s}(\alpha, \beta)$. Then by Theorem 2.1 , we have

$$
\begin{aligned}
& \sum_{n=1}^{\infty} \frac{d_{n}}{p(1-\alpha \beta) a_{p, 1}}\left|a_{n, 1}\right| \leq 1 . \\
& \sum_{n=1}^{\infty} \frac{d_{n}}{p(1-\alpha \beta) a_{p, 2}}\left|a_{n, 2}\right| \leq 1 .
\end{aligned}
$$

Employing the technique used earlier by Schild and Silverman [32],we need to find smallest $\delta$ such that

$$
\sum_{n=1}^{\infty} \frac{(n-p+p \alpha \delta) C_{b}^{s}(n)}{p(1-\alpha \delta) a_{p, 1} a_{p, 2}}\left|a_{n, 1}\right|\left|a_{n, 2}\right| \leq 1
$$

By Cauchy-Schwarz inequality, we have

$$
\sum_{n=1}^{\infty} \frac{d_{n}}{p(1-\alpha \beta) \sqrt{a_{p, 1} a_{p, 2}}} \sqrt{\left|a_{n, 1}\right|\left|a_{n, 2}\right|} \leq 1
$$

then

$$
\frac{(n-p+p \alpha \delta) C_{b}^{s}(n)\left|a_{n, 1}\right|\left|a_{n, 2}\right|}{p(1-\alpha \delta) a_{p, 1} a_{p, 2}} \leq \frac{d_{n}}{p(1-\alpha \beta) \sqrt{a_{p, 1} a_{p, 2}}} \sqrt{\left|a_{n, 1}\right|\left|a_{n, 2}\right|} .
$$

Hence that,

$$
\sqrt{\left|a_{n, 1}\right|\left|a_{n, 2}\right|} \leq \frac{d_{n}(1-\alpha \delta) \sqrt{a_{p, 1} a_{p, 2}}}{(n-p+p \alpha \delta) C_{b}^{s}(n)(1-\alpha \beta)} .
$$

we know that

$$
\sqrt{\left|a_{n, 1}\right|\left|a_{n, 2}\right|} \leq \frac{p(1-\alpha \beta) \sqrt{a_{p, 1} a_{p, 2}}}{d_{n}} .
$$

from (27) and (28), we have

$$
\frac{p(1-\alpha \beta)}{d_{n}} \leq \frac{d_{n}(1-\alpha \delta)}{(n-p+p \alpha \delta) C_{b}^{s}(n)(1-\alpha \beta)} .
$$

It follows that

$$
\delta=\frac{1}{\alpha}\left(1-\frac{n p(1-\alpha \beta)^{2} C_{b}^{s}(n)}{p^{2}(1-\alpha \beta)^{2} C_{b}^{s}(n)+d_{n}^{2}}\right) .
$$

Now defining a function $\Psi(n)$ by

$$
\Psi(n)=\frac{1}{\alpha}\left(1-\frac{n p(1-\alpha \beta)^{2} C_{b}^{s}(n)}{p^{2}(1-\alpha \beta)^{2} C_{b}^{s}(n)+d_{n}^{2}}\right),(n \geq 1),
$$

we observe that $\Psi(n)$ is an increasing function of $n$. We thus, conclude that

$$
\delta=\Psi(1)=\frac{1}{\alpha}\left(1-\frac{p(1-\alpha \beta)^{2} C_{b}^{s}(1)}{p^{2}(1-\alpha \beta)^{2} C_{b}^{s}(1)+d_{1}^{2}}\right)
$$

which completes the proof.

Theorem 4.2. For functions $f_{1}(z) \in \mathcal{M}_{b}^{s}(\alpha, \beta)$ and $f_{2}(z) \in \mathcal{M}_{b}^{s}(\alpha, \gamma)$, then $\left(f_{1} * f_{2}\right)(z) \in \mathcal{M}_{b}^{s}(\alpha, \zeta)$ where

$$
\zeta \leq \frac{(1+\alpha \beta)(1+\alpha \gamma) C_{b}^{s}(1)-(1-\alpha \beta)(1-\alpha \gamma)}{\alpha\left[(1-\alpha \beta)(1-\alpha \gamma)+(1+\alpha \beta)(1+\alpha \gamma) C_{b}^{s}(1)\right]}
$$


where

$$
\begin{aligned}
& d_{n}(p, \alpha, \beta)=[n-p(1-\alpha \beta)], \\
& d_{n}(p, \alpha, \gamma)=[n-p(1-\alpha \gamma)],
\end{aligned}
$$

and

$$
d_{n}(p, \alpha, \zeta)=[n-p(1-\alpha \zeta)] .
$$

Proof. For the function

$$
f_{1}(z)=a_{p, 1} z^{-p}+\sum_{n=1}^{\infty}\left|a_{n, 1}\right| z^{n-p} \in \mathcal{M}_{b}^{s}(\alpha, \beta)
$$

and

$$
f_{2}(z)=a_{p, 2} z^{-p}+\sum_{n=1}^{\infty}\left|a_{n, 2}\right| z^{n-p} \in \mathcal{M}_{b}^{s}(\alpha, \gamma)
$$

then by Theorem 2.1, we have

$$
\sum_{n=1}^{\infty} \frac{d_{n}(p, \alpha, \beta) C_{b}^{s}(n)}{p(1-\alpha \beta) a_{p, 1}}\left|a_{n, 1}\right| \leq 1
$$

and

$$
\sum_{n=1}^{\infty} \frac{d_{n}(p, \alpha, \gamma) C_{b}^{s}(n)}{p(1-\alpha \gamma) a_{p, 2}}\left|a_{n, 2}\right| \leq 1
$$

where

$$
d_{n}(p, \alpha, \beta)=[n-p(1-\alpha \beta)] \quad \text { and } \quad d_{n}(p, \alpha, \gamma)=[n-p(1-\alpha \gamma)] .
$$

Since $\left(f_{1} * f_{2}\right)(z) \in \mathcal{M}_{b}^{s}(\lambda, \alpha, \zeta)$, and again by Theorem 2.1 , we have

$$
\sum_{n=1}^{\infty} \frac{d_{n}(p, \alpha, \zeta) C_{b}^{s}(n)}{p(1-\alpha \zeta) a_{p, 1} a_{p, 2}}\left|a_{n, 1}\right|\left|a_{n, 2}\right| \leq 1
$$

Applying Cauchy-Schwarz inequality, we have

$$
\sum_{n=1}^{\infty} \frac{C_{b}^{s}(n) \sqrt{d_{n}(p, \alpha, \beta) d_{n}(p, \alpha, \gamma)}}{p \sqrt{(1-\alpha \beta)(1-\alpha \gamma) a_{p, 1} a_{p, 2}}} \sqrt{\left|a_{n, 1}\right|\left|a_{n, 2}\right|} \leq 1 .
$$

From(31)and (32), we have

$$
\begin{aligned}
& \frac{d_{n}(p, \alpha, \zeta) C_{b}^{s}(n)}{p(1-\alpha \zeta) a_{p, 1} a_{p, 2}}\left|a_{n, 1}\right|\left|a_{n, 2}\right| \leq \frac{C_{b}^{s}(n)}{p} \frac{\sqrt{d_{n}(p, \alpha, \beta) d_{n}(p, \alpha, \gamma)}}{\sqrt{(1-\alpha \beta)(1-\alpha \gamma) a_{p, 1} a_{p, 2}}} \sqrt{\left|a_{n, 1}\right|\left|a_{n, 2}\right|} \\
& \sqrt{\left|a_{n, 1}\right|\left|a_{n, 2}\right|} \leq \frac{\sqrt{d_{n}(p, \alpha, \beta) d_{n}(p, \alpha, \gamma)}}{\sqrt{(1-\alpha \beta)(1-\alpha \gamma)}} \frac{(1-\alpha \zeta) \sqrt{a_{p, 1} a_{p, 2}}}{d_{n}(p, \alpha, \zeta)}
\end{aligned}
$$

We know that

$$
\sqrt{\left|a_{n, 1}\right|\left|a_{n, 2}\right|} \leq \frac{p \sqrt{(1-\alpha \beta)(1-\alpha \gamma) a_{p, 1} a_{p, 2}}}{C_{b}^{s}(n) \sqrt{d_{n}(p, \alpha, \beta) d_{n}(p, \alpha, \gamma)}}
$$

from equation (33) and (34), we have

$$
\begin{aligned}
& \frac{p \sqrt{(1-\alpha \beta)(1-\alpha \gamma)}}{C_{b}^{s}(n) \sqrt{d_{n}(p, \alpha, \beta) d_{n}(p, \alpha, \gamma)}} \leq \frac{\sqrt{d_{n}(p, \alpha, \beta) d_{n}(p, \alpha, \gamma)}}{\sqrt{(1-\alpha \beta)(1-\alpha \gamma)}} \frac{(1-\alpha \zeta)}{d_{n}(p, \alpha, \zeta)} \\
& \zeta \leq \frac{1}{\alpha}\left[\frac{C_{b}^{s}(n) d_{n}(p, \alpha, \beta) d_{n}(p, \alpha, \gamma)-p(n-p)(1-\alpha \beta)(1-\alpha \gamma)}{d_{n}(p, \alpha, \beta) d_{n}(p, \alpha, \gamma) C_{b}^{s}(n)+p^{2}(1-\alpha \beta)(1-\alpha \gamma)}\right] .
\end{aligned}
$$


Now defining a function $\Psi(n)$ by

$$
\Psi(n)=\frac{1}{\alpha}\left[1-\frac{n p(1-\alpha \beta)(1-\alpha \gamma)}{d_{n}(p, \alpha, \beta) d_{n}(p, \alpha, \gamma) C_{b}^{s}(n)+p^{2}(1-\alpha \beta)(1-\alpha \gamma)}\right],(n \geq 1),
$$

we observe that $\Psi(n)$ is an increasing function of $n$. We thus, conclude that

$$
\zeta \leq \frac{(1+\alpha \beta)(1+\alpha \gamma) C_{b}^{s}(1)-(1-\alpha \beta)(1-\alpha \gamma)}{\alpha\left[(1-\alpha \beta)(1-\alpha \gamma)+(1+\alpha \beta)(1+\alpha \gamma) C_{b}^{s}(1)\right]}
$$

which completes the proof.

Theorem 4.3. Let the functions $f_{j}(z)(j=1,2)$ defined by

$$
f_{j}(z)=a_{p, i} z^{-p}+\sum_{n=1}^{\infty}\left|a_{n, j}\right| z^{n-p} \quad(j=1,2)
$$

be in the class $\mathcal{M}_{b}^{s}(\alpha, \beta, \rho)$ then the function $h(z)$ defined by

$$
h(z)=\left(a_{p, 1}+a_{p, 2}\right) z^{-p}+\sum_{n=1}^{\infty}\left(\left|a_{n, 1}\right|^{2}+\left|a_{n, 2}\right|^{2}\right) z^{n-p}
$$

belongs to the class $\mathcal{M}_{b}^{s}(\alpha, \gamma, \rho)$ where

$$
\gamma \leq \frac{1}{\alpha}\left(\frac{c_{1}^{2}-2 p(1-p)(1-\alpha \beta)^{2} C_{b}^{s}(1)+p \rho}{c_{1}^{2}+2 p^{2}(1-\alpha \beta)^{2}\left(C_{b}^{s}(1)-\rho\right)}\right) .
$$

Proof. Noting that

$$
\sum_{n=1}^{\infty}\left[\frac{c_{n}}{p(1-\alpha \beta)}\right]^{2}\left|a_{n, j}\right|^{2} \leq\left[\sum_{n=1}^{\infty} \frac{c_{n}}{p(1-\alpha \beta)}\left|a_{n, j}\right|\right]^{2} \leq 1,(j=1,2) .
$$

where

$$
c_{n}=[n-p(1-\alpha \beta)] C_{b}^{s}(n)+p(1-\alpha \beta) \rho^{n}
$$

For $f_{j}(z) \in \mathcal{M}_{b}^{s}(\alpha, \beta, \rho)(j=1,2)$, we have

$$
\sum_{n=1}^{\infty} \frac{1}{2}\left[\frac{c_{n}}{p(1-\alpha \beta)}\right]^{2}\left(\left|a_{n, 1}\right|^{2}+\left|a_{n, 2}\right|^{2}\right) \leq 1
$$

Therefore we have to find the largest $\gamma$ such that

$$
\sum_{n=1}^{\infty}\left[\frac{[n-p(1-\alpha \gamma)] C_{b}^{s}(n)+p(1-\alpha \gamma) \rho^{n}}{p(1-\alpha \gamma)}\right]\left(\left|a_{n, 1}\right|^{2}+\left|a_{n, 2}\right|^{2}\right) \leq 1,(n \geq 1)
$$

From equation (41) and (40) we have

$$
\begin{aligned}
& {\left[\frac{[n-p(1-\alpha \gamma)] C_{b}^{s}(n)+p(1-\alpha \gamma) \rho^{n}}{p(1-\alpha \gamma)}\right] \leq \frac{1}{2}\left[\frac{c_{n}}{p(1-\alpha \beta)}\right]^{2},(n \geq 1) .} \\
& \gamma \leq \frac{1}{\alpha}\left(\frac{c_{n}^{2}-2 p(n-p)(1-\alpha \beta)^{2} C_{b}^{s}(n)+p \rho^{n}}{c_{n}^{2}+2 p^{2}(1-\alpha \beta)^{2}\left(C_{b}^{s}(n)-\rho^{n}\right)}\right),(n \geq 1) .
\end{aligned}
$$

Now defining a function $\Psi(n)$ by

$$
\Psi(n)=\frac{1}{\alpha}\left(\frac{c_{n}^{2}-2 p(n-p)(1-\alpha \beta)^{2} C_{b}^{s}(n)+p \rho^{n}}{c_{n}^{2}+2 p^{2}(1-\alpha \beta)^{2}\left(C_{b}^{s}(n)-\rho^{n}\right)}\right),(n \geq 1)
$$

we observe that $\Psi(n)$ is an increasing function of $n$. We thus conclude that

$$
\gamma \leq \frac{1}{\alpha}\left(\frac{c_{1}^{2}-2 p(1-p)(1-\alpha \beta)^{2} C_{b}^{s}(1)+p \rho}{c_{1}^{2}+2 p^{2}(1-\alpha \beta)^{2}\left(C_{b}^{s}(1)-\rho\right)}\right) .
$$




\section{Closure properties}

In this section, we consider integral transforms of functions in the class $\mathcal{M}_{b}^{s}(\alpha, \beta)$.

Theorem 5.1. Let the function $f(z)$ given by (1) be in $\mathcal{M}_{b}^{s}(\alpha, \delta)$. Then the integral operator

$$
F(z)=c \int_{0}^{1} u^{c+p-1} f(u z) d u, \quad(0<u \leq 1, \quad 0<c<\infty),
$$

is in $M_{p}(\alpha, \delta)$ where

$$
\delta=\frac{1}{\alpha}\left(\frac{(c+n)(n-p+p \alpha \beta)-c(n-p)(1-\alpha \beta)}{(c+n)(n-p+p \alpha \beta)+c p(1-\alpha \beta)}\right) .
$$

Proof. Let $f(z) \in \mathcal{M}_{b}^{s}(\alpha, \beta)$. Then

$$
\begin{aligned}
F(z) & =c \int_{0}^{1} u^{c+p-1} f(u z) d u \\
& =c \int_{0}^{1}\left(\frac{u^{c-1} a_{p}}{z^{p}}+\sum_{n=1}^{\infty} a_{n} u^{n+c-1} z^{n-p}\right) d u \\
& =\frac{a_{p}}{z^{p}}+\sum_{n=1}^{\infty}\left(\frac{c}{c+n}\right) a_{n} z^{n-p} .
\end{aligned}
$$

It is sufficient to show that

$$
\sum_{n=1}^{\infty} \frac{(n-p+p \alpha \delta)}{p(1-\alpha \delta) a_{p}}\left(\frac{c}{c+n}\right) a_{n} \leq 1 .
$$

Since $f \in \mathcal{M}_{b}^{s}(\alpha, \beta)$, we have

$$
\sum_{n=1}^{\infty} \frac{(n-p+p \alpha \beta)}{p(1-\alpha \beta) a_{p}} a_{n} \leq 1 .
$$

Note that (45) is satisfied if

$$
\frac{(n-p+p \alpha \delta)}{p(1-\alpha \delta) a_{p}}\left(\frac{c}{c+n}\right) \leq \frac{(n-p+p \alpha \beta)}{p(1-\alpha \beta) a_{p}} .
$$

Rewriting the inequality, we have

$$
c(n-p+p \alpha \delta)(1-\alpha \beta) \leq(c+n)(n-p+p \alpha \beta)(1-\alpha \delta) .
$$

Solving for $\delta$, we have

$$
\begin{aligned}
& \delta \leq \frac{1}{\alpha}\left(\frac{(c+n)(n-p+p \alpha \beta)-c(n-p)(1-\alpha \beta)}{(c+n)(n-p+p \alpha \beta)+c p(1-\alpha \beta)}\right)=F(n) . \\
& F(n+1)-F(n)=\frac{1}{\alpha}\left(\frac{c(1-\alpha \beta)}{[c+n-p(1-\alpha \beta)][c+n+1-p(1-\alpha \beta)]}\right)>0,
\end{aligned}
$$

for each $n$. Hence, $F(n)$ is an increasing function of $n$, which yields the desired result(44).

Theorem 5.2. Let $f(z)$ given by (1), be in $\mathcal{M}_{b}^{s}(\alpha, \beta)$. Then

$$
F(z)=\frac{1}{c}\left[(c+p) f(z)+z f^{\prime}(z)\right]=\frac{a_{p}}{z^{p}}+\sum_{n=1}^{\infty} \frac{c+n}{c} a_{n} z^{n-p}, \quad c>0,
$$

is in $\mathcal{M}_{b}^{s}(\alpha, \beta)$ for $|z| \leq r(\alpha, \beta, \delta)$ where

$$
r(\alpha, \beta, \delta)=\inf _{n}\left(\frac{c(1-\alpha \delta)\{n-p+p \alpha \beta\}}{(1-\alpha \beta)(c+n)\{n-p+p \alpha \delta\}}\right)^{1 / n}, \quad n=1,2,3, \ldots
$$


Proof. Let $w=\left\{\frac{-z\left(\mathcal{J}_{b}^{s} f(z)\right)^{\prime}}{p \alpha\left(\mathcal{J}_{b}^{s} f(z)\right)}\right\}$. Then it is sufficient to show that

$$
\left|\frac{w+p}{w-p+2 \delta}\right|<1
$$

A computation shows that this is satisfied if

$$
\sum_{n=1}^{\infty} \frac{(n-p+p \alpha \delta)}{p(1-\alpha \delta) a_{p}} \frac{c+n}{c} a_{n}|z|^{n} \leq 1
$$

Since $f \in \mathcal{M}_{b}^{s}(\alpha, \beta)$, by Theorem 2.1 , we have

$$
\sum_{n=1}^{\infty} \frac{(n-p+p \alpha \beta)}{p(1-\alpha \beta) a_{p}}\left|a_{n}\right| \leq 1
$$

The equation (48) is satisfied if

$$
\sum_{n=1}^{\infty} \frac{(n-p+p \alpha \delta)(c+n)}{c(1-\alpha \delta)}|z|^{n} \leq \sum_{n=1}^{\infty} \frac{(n-p+p \alpha \beta)}{(1-\alpha \beta)}
$$

A simple computation yields, the inequality asserted in equation (47).

Theorem 5.3. (Arithmetic Mean) Let the functions $f_{i}(z)(i=1,2, \ldots \mu)$ defined by

$$
f_{i}(z)=\frac{a_{p, i}}{z^{p}}+\sum_{n=1}^{\infty} a_{n, i} z^{n-p}, \quad\left(a_{n, i} \geq 0, i=1,2 \ldots, \mu, n \geq 1\right)
$$

be in the class $\mathcal{M}_{b}^{s}(\alpha, \beta, \rho)$. Then the arithmetic mean of $f_{i}(z)(i=1,2, \ldots \mu)$ defined by

$$
h(z)=\frac{1}{\mu} \sum_{n=1}^{\mu} f_{i}(z)
$$

is also in the class $\mathcal{M}_{b}^{s}(\alpha, \beta, \rho)$.

proof Since $f_{i}(z) \in \mathcal{M}_{b}^{s}(\alpha, \beta, \rho)(i=1,2, \ldots \mu)$, then by using Theorem 2.3 , we have

$$
\begin{aligned}
& \sum_{n=1}^{\infty}\left[d_{n}+p(1-\alpha \beta) \rho^{n}\right]\left(\frac{1}{\mu} \sum_{i=1}^{\mu} a_{n, i}\right) \\
= & \frac{1}{\mu} \sum_{i=1}^{\mu}\left(\sum_{n=1}^{\infty}\left[d_{n}+p(1-\alpha \beta) \rho^{n}\right] a_{n, i}\right) \\
\leq & \frac{1}{\mu} \sum_{i=1}^{\mu} p(1-\alpha \beta) \\
\leq & p(1-\alpha \beta)
\end{aligned}
$$

which in view of Theorem 2.3, again implies that $h(z) \in \mathcal{M}_{b}^{s}(\alpha, \beta, \rho)$ and so the proof is complete.

Theorem 5.4. (Weighted Mean) Let the functions $f_{i}(z)(i=1,2)$ defined by

$$
f_{i}(z)=\frac{a_{p, i}}{z^{p}}+\sum_{n=1}^{\infty} a_{n, i} z^{n-p}, \quad\left(a_{n, i} \geq 0, i=1,2\right)
$$

be in the class $\mathcal{M}_{b}^{s}(\alpha, \beta, \rho)$. Then the weighted mean of $f_{i}(z)(i=1,2)$ defined by

$$
W_{c}(z)=\frac{1}{2}\left[(1-c) f_{1}(z)+(1+c) f_{2}(z)\right]
$$

is also in the class $\mathcal{M}_{b}^{s}(\alpha, \beta, \rho)$. 
proof Since

$$
f_{i}(z)=\frac{a_{p, i}}{z^{p}}+\sum_{n=1}^{\infty} a_{n, i} z^{n-p} \in \mathcal{M}_{b}^{s}(\alpha, \beta, \rho)
$$

for $\left(a_{n, i} \geq 0, i=1,2\right)$ and by (49) we have,

$$
W_{c}(z)=\left(a_{p, 1}+a_{p, 2}\right) z^{-p}+\sum_{n=1}^{\infty} \frac{1}{2}\left[(1-c) a_{n, 1}+(1+c) a_{n, 2}\right] z^{n-p} .
$$

By using Theorem 2.3,

$$
\sum_{n=1}^{\infty} \frac{\left[d_{n}+p(1-\alpha \beta) \rho^{n}\right]}{p(1-\alpha \beta)}\left|a_{n, 1}\right| \leq 1
$$

and

$$
\sum_{n=1}^{\infty} \frac{\left[d_{n}+p(1-\alpha \beta) \rho^{n}\right]}{p(1-\alpha \beta)}\left|a_{n, 2}\right| \leq 1 .
$$

Using 50and 51 in 49 , we get

$$
\begin{aligned}
W_{c}(z) & =\frac{1}{2}(1-c) p(1-\alpha \beta)+\frac{1}{2}(1+c) p(1-\alpha \beta) \\
& \leq p(1-\alpha \beta)
\end{aligned}
$$

Therefore $W_{c}(z) \in \mathcal{M}_{b}^{s}(\alpha, \beta, \rho)$, which completes the proof.

\section{Concluding remarks}

If $b=1, s=\nu(\nu>-1)$ the operator $\mathcal{J}_{b}^{s}$ turns into Libera-Bernardi integral operator $\mathcal{L}_{\nu}$ and if $b=\sigma(\sigma>0), s=1$ the operator $\mathcal{J}_{b}^{s}$ turns into Jung-Kim-Srivastava integral operator $\mathcal{I}_{\sigma}$. So, various other interesting corollaries and consequences of our main results can be derived similarly. The details involved may be left as an exercise for the interested reader.

\section{Acknowledgements}

The authors record their sincere thanks to the referees and Prof.G.Murugusundaramoorthy, SAS, VIT University, Vellore for his valuable suggestions during the preparation of this paper in the present form.

\section{References}

[1] J. W. Alexander, "Functions which map the interior of the unit circle upon simple regions", Ann. Math. Ser.2, 17 (1915), pp. $12-22$.

[2] M. K. Aouf, " On a certain class of meromorphic univalent functions with positive coefficients", Rend. Mat. Appl. 7 (11), (1991), no. 2, pp.209-219.

[3] M. K. Aouf and H. M. Hossen, "New criteria for meromorphic p-valent starlike functions", Tsukuba J. Math. 17(1993),pp. 481-486.

[4] M. K. Aouf, H. M. Hossen and H. E. Elattar, "A certain class of meromorphic multivalent functions with positive and fixed second coefficients", Punjab Univ. J. Math. 33(2000), pp.115-124.

[5] S. K. Bajpai," A note on a class of meromorphic univalent functions", Rev. Roumaine Math. Pures Appl. 22 (1977), no. 3, pp.295-297.

[6] S. D. Bernardi, "Convex and starlike univalent functions", Trans. Amer. Math. Soc., 135 (1969), pp.429-446.

[7] N. E. Cho, S. H. Lee and S. Owa, " A class of meromorphic univalent functions with positive coefficients", Kobe J. Math., 4 (1987), no. 1, pp.43-50. 
[8] J. Choi and H. M. Srivastava, "Certain families of series associated with the Hurwitz-Lerch Zeta function", Appl. Math. Comput., 170 (2005), pp.399-409.

[9] J. Clunie, " On meromorphic schlicht functions", J. London Math. Soc., 34 (1959), pp.215-216.

[10] C. Ferreira and J. L. Lopez, "Asymptotic expansions of the Hurwitz-Lerch Zeta function", J. Math. Anal. Appl., 298(2004), pp.210-224.

[11] T. M. Flett, "The dual of an inequality of Hardy and Littlewood and some related inequalities", J. Math. Anal. Appl., 38(1972), pp.746-765

[12] M. Garg, K. Jain and H. M. Srivastava, "Some relationships between the generalized Apostol-Bernoulli polynomials and Hurwitz-Lerch Zeta functions", Integral Transform. Spec. Funct., 17(2006), pp.803-815.

[13] S.-D. Lin and H. M. Srivastava, "Some families of the Hurwitz-Lerch Zeta functions and associated fractional derivative and other integral representations", Appl. Math. Comput., 154(2004), pp.725-733.

[14] S.-D. Lin, H. M. Srivastava and P.-Y. Wang, "Some expansion formulas for a class of generalized Hurwitz-Lerch Zeta functions", Integral Transform. Spec. Funct., 17(2006), pp.817-827.

[15] Joshi, S. B. and M. K. Aouf, " Meromorphic multivalent functions with positive and fixed second coefficients", Kyungpook Math. J., 35 (1995), pp.163-169.

[16] Joshi, S. B. and H. M. Srivastava, "A certain family of meromorphically multivalent functions", Comput. Math. Appl. 38, no. 3-4,(1999) pp.201-211.

[17] I. B. Jung, Y. C. Kim AND H. M. Srivastava, "The Hardy space of analytic functions associated with certain oneparameter families of integral operators", J. Math. Anal. Appl., 176(1993), pp.138-147.

[18] Kulkarni, S. R., U. H. Naik and H. M. Srivastava, "A certain class of meromorphically p-valent quasi-convex functions", PanAmer. Math. J., 8, no. 1,(1998) pp.57-64.

[19] R. J. Libera, "Some classes of regular univalent functions", Proc. Amer. Math. Soc., 16 (1969), pp.755-758.

[20] J-L. Liu, "Some Inclusion Properties for Certain Subclass of Meromorphically Multivalent Functions Involving the Srivastava-Attiya Operator", Tamsui Oxford Journal of Information and Mathematical Sciences. 28(3) (2012) 267-279.

[21] J.-L. Liu, H.M. Srivastava, "Classes of meromorphically multivalent functions associated with the generalized hypergeometric function", Math. Comput. Modelling 39 (2004) pp.21-34.

[22] J.-L. Liu, H.M. Srivastava, "Subclasses of meromorphically multivalent functions associated with a certain linear operator", Math. Comput. Modelling 39 (2004) pp.35-44.

[23] M. L. Mogra, T. R. Reddy, O. P. Juneja, "Meromorphic univalent functions with positive coefficients", Bull. Austral. Math. Soc., 32 (1985), pp.161-176.

[24] M. Darus, "Meromorphic functions with positive coefficients", Int. J. Math. Math. Sci., 4 (2004), pp.319-324.

[25] Montel. P., "Lecons sur les Fonctions Univalentes ou Multivalentes", Gauthier-Villars, Paris (1933).

[26] Z. Nehari, E. Netanyahu," On the coefficients of meromorphic schlicht functions", Proc. Amer. Math. Soc., 8 (1957), pp.15-23.

[27] Owa, S., and N. N. Pascu, " Coefficient inequalities for certain classes of meromorphically starlike and meromorphically convex functions", J. Inequal. Pure Appl. Math., 4, no. 1, (2003) Article 17, pp.1-6.

[28] Ch. Pommerenke," ber einige Klassen meromorpher schlichter Funktionen”, Math. Z.(German), 78 (1962), pp.263-284.

[29] Ch. Pommerenke, " On meromorphic starlike functions", Pacific J. Math., 13 (1963), pp.221-235.

[30] Raina, R. K. and H. M. Srivastava, " A new class of meromorphically multivalent functions with applications to generalized hypergeometric functions", Math. Comput. Modelling, 43,(2006) pp.350-356.

[31] W. C. Royster," Meromorphic starlike multivalent functions", Trans. Amer. Math. Soc., 107 (1963), pp.300-308.

[32] A. Schild and H. Silverman, "Convolution of univalent functions with negative coefficients", Ann. Univ. Mariae-Curie Sk lodowska, Sect.A 29 (1975), pp.99-107. 
[33] H. M. Srivastava, S. Owa (eds.), " Current Topics in Analytic Function Theory", World Scientific Publishing, New Jersey, 1992.

[34] H. M. Srivastava and A. A. Attiya, "An integral operator associated with the Hurwitz-Lerch Zeta function and differential subordination", Integral Transform. Spec. Funct., 18(2007), pp.207-216.

[35] H. M. Srivastava H. M. Hossen and M. K. Aouf, "A unified presentation of some classes of meromorphically multivalent functions", Comput. Math. Appl., 38(1999), pp.63-70.

[36] B. A. Uralegaddi, M. D. Ganigi, "A certain class of meromorphically starlike functions with positive coefficients", Pure Appl. Math. Sci., 26(1987), pp.75-81.

[37] B. A. Uralegaddi and C. Somanatha, " Certain differential operators for meromorphic functions", Houston J. Math., 17 (1991), no. 2, pp.279-284.

[38] K.Vijaya,G.Murugusundaramoorthy and P.Kathiravan " Multivalently meromorphic functions associated with convolution structure", Acta Universitatis Apulensis, No. 34(2013) pp. 247-263

[39] Yang, D.G., "Subclasses of meromorphic p-valent convex functions", J. Math. Res. Exposition 20(2000), pp.215-219. 